\title{
Prospective Analysis in GIST Patients on the Role of Alpha-1 Acid Glycoprotein in Imatinib Exposure
}

\author{
Sander Bins ${ }^{1} \cdot$ Karel Eechoute $^{1} \cdot$ Jacqueline S. L. Kloth $^{1} \cdot$ Femke M. de Man $^{1}$ • \\ Astrid W. Oosten ${ }^{1} \cdot$ Peter de Bruijn $^{1} \cdot$ Stefan Sleijfer $^{1} \cdot$ Ron H. J. Mathijssen ${ }^{1}$
}

Published online: 26 July 2016

(c) The Author(s) 2016. This article is published with open access at Springerlink.com

\begin{abstract}
Background For imatinib, a relationship between systemic exposure and clinical outcome has been suggested. Importantly, imatinib concentrations are not stable and decrease over time, for which several mechanisms have been suggested. In this study, we investigated if a decrease in alpha-1 acid glycoprotein (AGP) is the main cause of the lowering in imatinib exposure over time.

Methods We prospectively measured imatinib trough concentration $\left(C_{\mathrm{min}}\right)$ values in 28 patients with gastrointestinal stromal tumours, at 1,3 and 12 months after the start of imatinib treatment. At the same time points, AGP levels were measured.

Results Overall, imatinib $C_{\min }$ and AGP levels were correlated $\left(r^{2}=0.656 ; P<0.001\right)$. However, AGP levels did not fluctuate significantly over time, nor did the change in AGP levels correlate with the change in the imatinib $C_{\text {min }}$. Conclusion We showed that systemic AGP levels are not likely to be a key player in the decrease in systemic imatinib exposure over time. As long as intra-individual changes in imatinib exposure remain unexplained, researchers should standardize the sampling times for imatinib in order to be able to assess the clinical applicability of therapeutic drug monitoring.
\end{abstract}

Ron H. J. Mathijssen

a.mathijssen@erasmusmc.nl

1 Department of Medical Oncology, Erasmus MC Cancer Institute, PO Box 5201, 3008 AE Rotterdam, The Netherlands

\section{Key Points}

The decrease in systemic imatinib exposure during the first months of treatment is not likely to be caused by increased clearance due to fluctuations in alpha-1 acid glycoprotein levels.

Since systemic imatinib exposure varies over time, pharmacokinetic sampling should be performed at standardized time points.

\section{Background}

Imatinib is one of the first tyrosine kinase inhibitors (TKIs) for which therapeutic drug monitoring (TDM) is deemed suitable in the treatment of both chronic myeloid leukaemia (CML) and gastrointestinal stromal tumours (GISTs). In CML, higher imatinib exposure has been found in patients with a treatment response [1-4], and imatinib trough concentration $\left(C_{\min }\right)$ values above $1000 \mathrm{ng} / \mathrm{mL}$ have been found to be predictive of higher response rates [1, 2]. For GIST, the target imatinib $C_{\min }$ has been established in a phase II study, in which patients with an imatinib $C_{\min }$ in the lowest quartile (i.e. below $1100 \mathrm{ng} / \mathrm{mL}$ ) had significantly worse progression-free survival (PFS), and it was suggested that this concentration should serve as a target $C_{\text {min }}$ [5]. Studies conducted in the context of routine care have shown that more than half of imatinib-treated patients do not reach that $C_{\min }$ [6-8]. In these studies performed in daily practice, the $C_{\min }$ was measured more than 3 months after the start of treatment, whereas in the study describing the threshold of $1100 \mathrm{ng} / \mathrm{mL}$, the imatinib $C_{\min }$ was 
established after 4 weeks of treatment. Meanwhile, it has been shown that imatinib clearance increases-and systemic concentrations therefore decrease-during the first 3 months of treatment $[9,10]$. Hence, it could be expected upfront that an even larger proportion of patients than the $25 \%$ in the phase II study would have a $C_{\min }$ below $1100 \mathrm{ng} / \mathrm{mL}$ when it was determined later than 3 months after the start of treatment, and doubts have been raised as to whether this threshold set at a time when systemic exposure has not yet stabilized is indeed the appropriate target imatinib $C_{\min }$ in patients with GIST [11]. Accordingly, in one of the more recent retrospective studies in GIST patients, a threshold of $760 \mathrm{ng} / \mathrm{mL}$ led to better prediction of the outcome [8]. In the same study population, however, the median PFS was longer for patients with a $C_{\min }>1100 \mathrm{ng} / \mathrm{mL}$ than for those with a $C_{\min }>760 \mathrm{ng} /$ $\mathrm{mL}$ (67 versus 56 months) [8].

Several mechanisms have been suggested to account for the reduction in systemic imatinib concentrations over time, the first being decreased absorption [9]. Alternatively, Chatelut et al. [12] proposed that systemic imatinib exposure decreases because of increased clearance rather than because of decreased absorption. As imatinib is predominantly bound to the acute-phase protein alpha-1 acid glycoprotein (AGP) [13-15], a reduction in AGP over time would lead to less protein-bound imatinib and therefore a larger proportion of free imatinib that could be metabolized or excreted [12]. According to this theory, it is assumed that a decrease in the tumour burden leads to a reduced inflammatory syndrome, which in turn causes lowering of AGP levels. The finding that changes in AGP levels over time correlate well with changes in imatinib concentrations seems to back this mechanism [16]. However, these data were analysed in retrospect and, more importantly, they were not collected in a structured manner, as imatinib concentrations and AGP levels were measured at separate time points. Additionally, AGP levels and imatinib concentrations were not assessed synchronously in that study. To firmly establish the influence of AGP levels on blood imatinib concentrations, this study aimed to prospectively assess the correlation between imatinib $C_{\min }$ values and AGP levels.

\section{Methods}

\subsection{Patients}

Adult patients with GIST in whom commencement of imatinib treatment was planned were eligible for inclusion in this study. The exclusion criteria were prior imatinib treatment within 3 months prior to the start of the study, major surgery within 2 weeks prior to the start of the study, use of potent cytochrome P450 (CYP) 3A inhibitors or inducers, and inability to give or understand informed consent. The study protocol was approved by the local institutional review board (protocol number MEC13-203). All procedures performed in this study were in accordance with the ethical standards of the institutional and/or national research committee and with the 1964 Helsinki Declaration and its later amendments or comparable ethical standards. Written informed consent was obtained from all individual participants included in the study.

\subsection{Study Procedures}

Pharmacokinetic sampling was performed at 1,3 and 12 months after the start of imatinib treatment. At each time point, two blood samples were collected in addition to the standard-of-care blood draw at scheduled outpatient visits. The first sample was collected in a glass tube containing lithium heparin as an anticoagulant, and was used to quantify the concentrations of imatinib and its main metabolite, CGP74588. This sample was processed to plasma within 30 minutes by centrifugation for $15 \mathrm{~min}$ at $2500 \times g\left(4{ }^{\circ} \mathrm{C}\right)$. Next, the plasma was transferred to polypropylene tubes and stored at $-70{ }^{\circ} \mathrm{C}$ until the time of analysis at the Laboratory of Translational Pharmacology, Erasmus MC Cancer Institute (Rotterdam, the Netherlands). The methods used for quantification of imatinib and CGP74588 concentrations have been described previously [17]. The second sample was collected in a serum-separating tube and processed to serum. Serum AGP levels were measured using an immunoturbidimetric assay on a Cobas Integra 800 (Roche Diagnostics GmbH, Mannheim, Germany) in accordance with the instructions of the manufacturer. Briefly, serum AGP was agglutinated with a polyclonal goat-antihuman AGP antibody. The amount of agglutination of the antigen-antibody complex was measured turbidimetrically.

\subsection{Statistical Considerations}

At least 24 patients had to be included to identify a rho value of 0.55 in a two-sided test with alpha $=0.05$ and power $=0.8$. Correlation was tested using Pearson's correlation, equality of two means was tested using $t$ tests and equality of more than two means was tested using one-way analysis of variance (ANOVA). $P$ values $<0.05$ were considered statistically significant. Descriptive statistics were performed using IBM SPSS Statistics version 21 software (SPSS, Chicago, IL, USA). All other statistical analyses were performed using GraphPad Prism 5.0 software (GraphPad Software, La Jolla, CA, USA). 


\section{Results}

\subsection{Baseline}

Between April 2013 and March 2015, 35 patients signed informed consent and were included in the study. Four patients were not evaluable because they stopped imatinib treatment within 3 months and therefore did not provide repetitive pharmacokinetic samples. In another three patients, only one $C_{\min }$ value was available because the patients had taken imatinib prior to the other sampling time points or because they had an imatinib concentration below the limit of quantification $(<20.0 \mathrm{ng} / \mathrm{mL})$. The baseline characteristics of the 28 evaluable patients are depicted in Table 1. Eight of the evaluable patients stopped treatment before the final sampling time point because of progressive disease $(n=3)$, cessation of neoadjuvant treatment $(n=4)$ or toxicity $(n=1)$.

Table 1 Baseline patient characteristics

\begin{tabular}{|c|c|}
\hline Characteristic & Patients, $n=28$ \\
\hline \multicolumn{2}{|l|}{ Age at start } \\
\hline Years & $69(10)$ \\
\hline \multicolumn{2}{|l|}{ Sex } \\
\hline Male & $16(57 \%)$ \\
\hline Female & $12(43 \%)$ \\
\hline \multicolumn{2}{|c|}{ WHO performance status } \\
\hline 0 & $12(43 \%)$ \\
\hline 1 & $13(46 \%)$ \\
\hline 2 & $1(4 \%)$ \\
\hline Unknown & $2(7 \%)$ \\
\hline \multicolumn{2}{|l|}{$c-K I T$ mutation } \\
\hline Wild type & $5(18 \%)$ \\
\hline Exon 9 & $6(21 \%)$ \\
\hline Exon 11 & $12(43 \%)$ \\
\hline Exon 13 & $3(11 \%)$ \\
\hline Unknown & $2(7 \%)$ \\
\hline \multicolumn{2}{|c|}{ Treatment setting } \\
\hline Neoadjuvant & $11(39 \%)$ \\
\hline Adjuvant & $5(18 \%)$ \\
\hline Palliative & $12(43 \%)$ \\
\hline \multicolumn{2}{|l|}{ Dose at start } \\
\hline $300 \mathrm{mg}$ QD & $1(4 \%)$ \\
\hline $400 \mathrm{mg}$ QD & $26(93 \%)$ \\
\hline $800 \mathrm{mg}$ QD & $1(4 \%)$ \\
\hline
\end{tabular}

All values are presented as $n(\%)$ or as mean (standard deviation) $c$-KIT KIT proto-oncogene receptor tyrosine kinase, $Q D$ once daily, WHO World Health Organization

\subsection{AGP Levels and Imatinib Concentrations}

A total of 73 imatinib trough concentration samples were obtained. In 69 cases, a blood sample for measurement of the AGP level was collected synchronically. The mean values of AGP, imatinib and CGP74588 at each time point are depicted in Table 2. At any of the three time points, the AGP levels in the five patients treated in the adjuvant setting did not differ significantly from those in the patients treated in the neoadjuvant or palliative settings (Fig. 1a).

\subsection{AGP Versus Imatinib}

Overall, AGP levels were significantly correlated with imatinib concentrations $\left(r^{2}=0.656 ; P<0.001\right.$; Fig. 2) and with the sum of imatinib and CGP74588 concentrations $\left(r^{2}=0.667 ; P<0.001\right)$. The correlation between imatinib concentrations and AGP levels was less strong in the 25 samples that were taken at the first time point after 30 days $\left(r^{2}=0.526 ; P<0.001\right.$; Fig. 3$)$ in comparison with the correlations assessed at the two later time points. The absolute difference in AGP levels between time points 1 and 2 was also significantly correlated with the absolute difference in imatinib concentrations between time points 1 and $2\left(r^{2}=0.381 ; P=0.002\right)$ and between time points 1 and $3\left(r^{2}=0.355 ; P=0.03\right)$. The relative differences in AGP levels and imatinib concentrations between time points were not significantly correlated. The geometric mean AGP levels did not differ significantly between the three time points $(P=0.141$; Fig. $1 b)$.

\section{Discussion}

In this prospective setting, imatinib pharmacokinetics were closely correlated with systemic AGP levels when all samples obtained at the three different time points were considered together $\left(r^{2}=0.656 ; P<0.001\right)$. Althoughat first sight- this appeared to be in line with the hypothesis that the increase in imatinib clearance is due to reduced systemic AGP levels $[14,16]$, the differences in AGP levels and imatinib $C_{\min }$ values between the time points were less strongly correlated. Moreover, the argument that AGP decreases during treatment and thereby contributes to increased imatinib clearance over time [12] did not seem to hold true, as we did not find substantial reductions in AGP levels during treatment $(P=0.141$; Fig. 1). Patients treated in the adjuvant setting even had a gradual increase in AGP levels, which contradicted the theory that AGP levels are initially elevated because of an inflammatory syndrome directly after tumour surgery and decline over time when the surgery effects resolve [12]. Even though the decrease in imatinib concentrations was 
Table 2 Analyses of the samples obtained at the different time points

\begin{tabular}{|c|c|c|c|c|}
\hline & Time point $1, n=25$ & Time point $2, n=25$ & Time point $3, n=19$ & Total, $n=69$ \\
\hline Actual time since start of imatinib [days] ${ }^{\mathrm{a}}$ & $30(3)$ & $97(30)$ & $364(20)$ & \\
\hline AGP level $[\mathrm{g} / \mathrm{L}]^{\mathrm{b}}$ & $0.97(0.85-1.10)$ & $0.81(0.69-0.94)$ & $0.89(0.78-1.00)$ & $0.89(0.82-0.96)$ \\
\hline Imatinib concentration $[\mathrm{ng} / \mathrm{mL}]^{\mathrm{b}}$ & 1457 (1155-1838) & 1305 (1001-1702) & 1193 (967-1472) & $1325(1158-1516)$ \\
\hline CGP74588 concentration $[\mathrm{ng} / \mathrm{mL}]^{\mathrm{b}}$ & $308(247-384)$ & $265(205-343)$ & 231 (179-299) & $270(235-309)$ \\
\hline Imatinib + CGP74588 concentration $[\mathrm{ng} / \mathrm{mL}]^{\mathrm{b}}$ & $1777(1420-2224)$ & 1578 (1217-2047) & 1439 (1165-1777) & $1606(1407-1833)$ \\
\hline Correlation between imatinib and $\mathrm{AGP}^{\mathrm{c}}$ & $0.526(P<0.001)$ & $0.839(P<0.001)$ & $0.411(P=0.003)$ & $0.656(P<0.001)$ \\
\hline
\end{tabular}

The three time points are the times at which sampling was scheduled according to the protocol, i.e. 30 days, 90 days and 365 days after the start of treatment

AGP alpha-1 acid glycoprotein

${ }^{a}$ Units of time are presented as mean (standard deviation)

${ }^{\mathrm{b}}$ Values are presented as geometric mean (95\% confidence interval)

c Correlations are depicted as $r^{2}(P$ value $)$

(a)

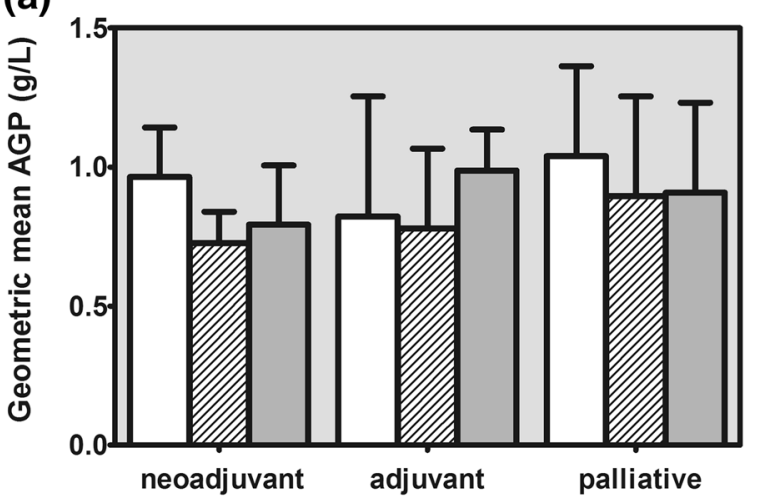

(b)

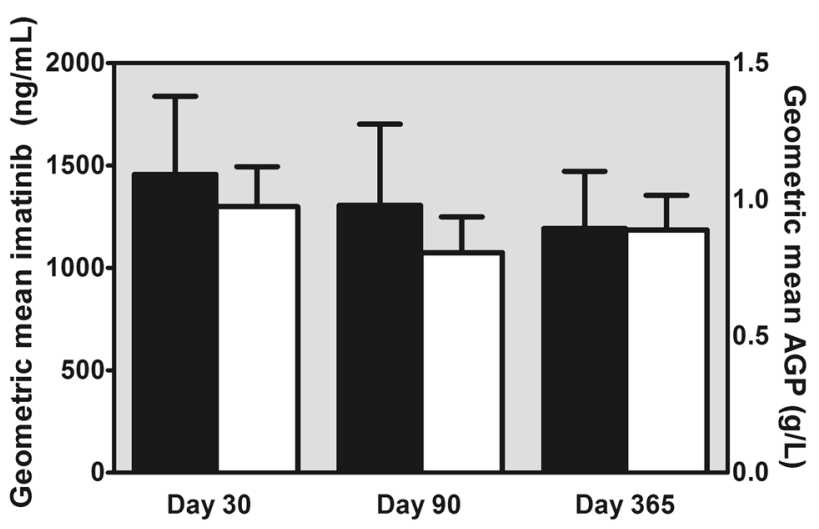

Fig. 1 a Geometric mean levels of alpha-1 acid glycoprotein (AGP) at 30 days (white bars), 90 days (striped bars) and 365 days (dark grey bars) from the start of treatment in each treatment setting. b Geometric mean trough concentrations of imatinib (black bars) and AGP (white bars) at each time point. The error bars represent the $95 \%$ confidence intervals

not as large as those published previously, the implication of our current findings is that the role of systemic AGP levels in the reduced systemic imatinib exposure over time

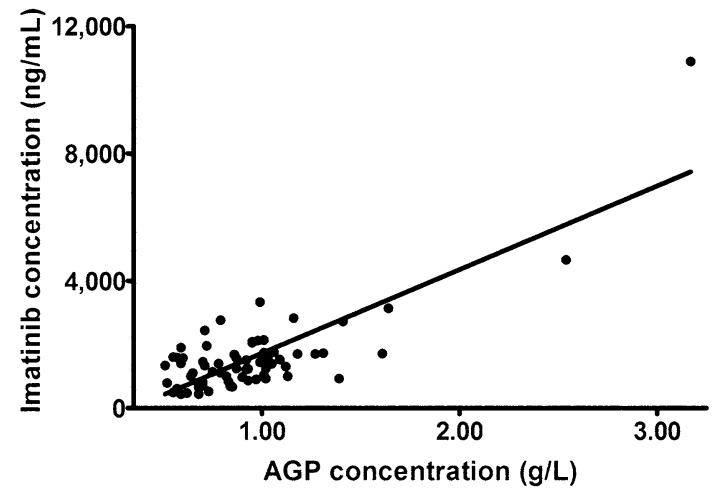

Fig. 2 Correlation between imatinib concentrations and alpha-1 acid glycoprotein (AGP) levels in all samples $(n=69)$

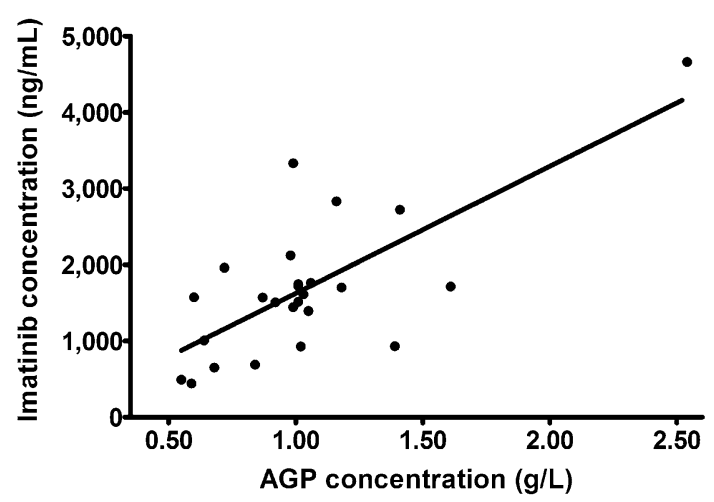

Fig. 3 Correlation between imatinib concentrations and alpha-1 acid glycoprotein (AGP) levels at 30 days $(n=25)$

is relatively small and that other factors, e.g. reduced bioavailability, likely have larger influences on systemic exposure. Still, AGP might seriously interfere with imatinib exposure in vivo, as extravascular AGP affects imatinib pharmacokinetics beyond the systemic circulation [15], and preclinical research has shown that the 
pharmacodynamic effects of imatinib are reduced in the presence of AGP [18-20]. Nonetheless, it remains questionable whether these extravascular effects can be used to determine the optimal dose for individual patients.

Unfortunately, the available evidence for individualized imatinib dosing in GIST patients is currently not robust, hampering assessment of the clinical relevance of TDM in GIST. Imatinib $C_{\min }$ values measured at different time points during treatment have previously been related to the clinical outcome [5, 8]. Also, as mentioned previously, because of the decrease in systemic imatinib concentrations over time, target $C_{\min }$ values after 1 month cannot be extrapolated into a dosing algorithm for the entire treatment period. Although it has been proposed that TDM be performed only after imatinib pharmacokinetics have stabilized after 3 months of treatment [11], whether or not an individual with GIST receives the proper treatment and dose would ideally become visible much earlier during treatment. For example, by using fludeoxyglucose $(18 \mathrm{~F})$ $\left[{ }^{18} \mathrm{~F}-\mathrm{FDG}\right]$ positron emission tomography [PET] as early as a few days after the start of treatment, it is possible to know whether or not a GIST patient is responding to treatment [21]. Either way, TDM in imatinib treatment can reach its full potential only when sampling times are standardized between research groups [22]. The sampling schedule employed in the current study could serve as a blueprint for larger studies because it incorporated $C_{\min }$ values at 1 month and at later time points, enabling assessment of long-term pharmacokinetic targets, which could subsequently be compared with the established target at 1 month. Alternatively, long-term pharmacokinetic targets could be derived from the target $C_{\min }$ at 1 month, using a formula that corrects for the parameters that contribute to the decrease in imatinib exposure. However, as the biological mechanism of this decreased exposure seems to be complex and multifactorial, the latter option to determine long-term pharmacokinetic targets is not likely to be computed soon. In parallel, other challenges in making TDM clinically usable will be to integrate the dosing range (300-800 mg daily) and the possible options in the case of insufficient concentrations (dose escalation or a treatment switch), but these are secondary to standardization of the sampling time points. Last, but certainly not least, it remains to be proven that imatinib TDM in GIST really translates into a better outcome in terms of either less toxicity or better anti-tumour effects.

\section{Conclusion}

We found that systemic AGP levels are not likely to be a key player in the decrease of systemic imatinib exposure over time. We believe that TDM is a very potent tool to improve personalized imatinib treatment, but it can flourish only if researchers ensure that their results are obtained in a standardized way.

\section{Compliance with Ethical Standards}

Funding This work was not supported by any external funding.

Conflict of interest S. Bins, K. Eechoute, J.S.L. Kloth, F.M. de Man, A.W. Oosten, P. de Bruijn, S. Sleijfer and R.H.J. Mathijssen declare that they have no conflicts of interest that are directly relevant to the content of this article.

Open Access This article is distributed under the terms of the Creative Commons Attribution-NonCommercial 4.0 International License (http://creativecommons.org/licenses/by-nc/4.0/), which permits any noncommercial use, distribution, and reproduction in any medium, provided you give appropriate credit to the original author(s) and the source, provide a link to the Creative Commons license, and indicate if changes were made.

\section{References}

1. Larson RA, Druker BJ, Guilhot F, O'Brien SG, Riviere GJ, Krahnke $\mathrm{T}$, et al. Imatinib pharmacokinetics and its correlation with response and safety in chronic-phase chronic myeloid leukemia: a subanalysis of the IRIS study. Blood. 2008;111(8):4022-8.

2. Picard S, Titier K, Etienne G, Teilhet E, Ducint D, Bernard MA, et al. Trough imatinib plasma levels are associated with both cytogenetic and molecular responses to standard-dose imatinib in chronic myeloid leukemia. Blood. 2007;109(8):3496-9.

3. Ishikawa Y, Kiyoi H, Watanabe K, Miyamura K, Nakano Y, Kitamura $\mathrm{K}$, et al. Trough plasma concentration of imatinib reflects BCR-ABL kinase inhibitory activity and clinical response in chronic-phase chronic myeloid leukemia: a report from the BINGO study. Cancer Sci. 2010;101(10):2186-92.

4. Zhong JS, Meng FY, Xu D, Zhou HS, Dai M. Correlation between imatinib trough concentration and efficacy in Chinese chronic myelocytic leukemia patients. Acta Haematol. 2012;127(4):221-7.

5. Demetri GD, Wang Y, Wehrle E, Racine A, Nikolova Z, Blanke $\mathrm{CD}$, et al. Imatinib plasma levels are correlated with clinical benefit in patients with unresectable/metastatic gastrointestinal stromal tumors. J Clin Oncol. 2009;27(19):3141-7.

6. Yoo C, Ryu M-H, Ryoo B-Y, Beck MY, Chang H-M, Lee J-L, et al. Changes in imatinib plasma trough level during long-term treatment of patients with advanced gastrointestinal stromal tumors: correlation between changes in covariates and imatinib exposure. Invest New Drugs. 2011;30(4):1703-8. doi:10.1007/ s10637-011-9633-5.

7. Lankheet NA, Knapen LM, Schellens JH, Beijnen JH, Steeghs N, Huitema AD. Plasma concentrations of tyrosine kinase inhibitors imatinib, erlotinib, and sunitinib in routine clinical outpatient cancer care. Ther Drug Monit. 2014;36(3):326-34.

8. Bouchet S, Poulette S, Titier K, Moore N, Lassalle R, Abouelfath A, et al. Relationship between imatinib trough concentration and outcomes in the treatment of advanced gastrointestinal stromal tumours in a real-life setting. Eur J Cancer. 2016;57:31-8.

9. Eechoute K, Fransson MN, Reyners AK, de Jong FA, Sparreboom A, van der Graaf WT, et al. A long-term prospective population pharmacokinetic study on imatinib plasma concentrations in GIST patients. Clin Cancer Res. 2012;18(20):5780-7. 
10. Judson I, Ma P, Peng B, Verweij J, Racine A, di Paola ED, et al. Imatinib pharmacokinetics in patients with gastrointestinal stromal tumour: a retrospective population pharmacokinetic study over time. EORTC Soft Tissue and Bone Sarcoma Group. Cancer Chemother Pharmacol. 2005;55(4):379-86.

11. Judson I. Therapeutic drug monitoring of imatinib-new data strengthen the case. Clin Cancer Res. 2012;18(20):5517-9.

12. Chatelut E, Gandia P, Gotta V, Widmer N. Long-term prospective population PK study in GIST patients [letter]. Clin Cancer Res. 2013;19(4):949.

13. Gambacorti-Passerini C, le Coutre P, Zucchetti M, D'Incalci M. Binding of imatinib by alpha(1)-acid glycoprotein. Blood. 2002;100(1):367-8 (author reply 378-9).

14. Widmer N, Decosterd LA, Csajka C, Leyvraz S, Duchosal MA, Rosselet A, et al. Population pharmacokinetics of imatinib and the role of alpha-acid glycoprotein. Br J Clin Pharmacol. 2006;62(1):97-112.

15. Zsila F, Fitos I, Bencze G, Keri G, Orfi L. Determination of human serum alpha1-acid glycoprotein and albumin binding of various marketed and preclinical kinase inhibitors. Curr Med Chem. 2009;16(16):1964-77.

16. Mathijssen RH, de Bruijn P, Eechoute K, Sparreboom A. Longterm prospective population PK study in GIST patients-response. Clin Cancer Res. 2013;19(4):950.

17. Schiavon G, Eechoute K, Mathijssen RH, de Bruijn P, van der Bol JM, Verweij J, et al. Biliary excretion of imatinib and its active metabolite CGP74588 during severe hepatic dysfunction. J Clin Pharmacol. 2012;52(7):1115-20.

18. Beckmann S, Long T, Scheld C, Geyer R, Caffrey CR, Grevelding CG. Serum albumin and alpha-1 acid glycoprotein impede the killing of Schistosoma mansoni by the tyrosine kinase inhibitor imatinib. Int $\mathrm{J}$ Parasitol Drugs Drug Resist. 2014;4(3):287-95.

19. Gambacorti-Passerini C, Barni R, le Coutre P, Zucchetti M, Cabrita G, Cleris L, et al. Role of alpha1 acid glycoprotein in the in vivo resistance of human BCR-ABL(+) leukemic cells to the abl inhibitor STI571. J Natl Cancer Inst. 2000;92(20):1641-50.

20. Larghero J, Leguay T, Mourah S, Madelaine-Chambrin I, Taksin $\mathrm{AL}$, Raffoux E, et al. Relationship between elevated levels of the alpha 1 acid glycoprotein in chronic myelogenous leukemia in blast crisis and pharmacological resistance to imatinib (Gleevec) in vitro and in vivo. Biochem Pharmacol. 2003;66(10):1907-13.

21. Van den Abbeele AD, Gatsonis C, de Vries DJ, Melenevsky Y, Szot-Barnes A, Yap JT, et al. ACRIN 6665/RTOG 0132 phase II trial of neoadjuvant imatinib mesylate for operable malignant gastrointestinal stromal tumor: monitoring with 18F-FDG PET and correlation with genotype and GLUT4 expression. J Nucl Med. 2012;53(4):567-74.

22. Yu H, Steeghs N, Nijenhuis CM, Schellens JH, Beijnen JH, Huitema AD. Practical guidelines for therapeutic drug monitoring of anticancer tyrosine kinase inhibitors: focus on the pharmacokinetic targets. Clin Pharmacokinet. 2014;53(4):305-25. 